\title{
A NOTE ON WITHDRAWAL THROUGH A POINT SINK IN FLUID OF FINITE DEPTH
}

\author{
LAWRENCE K. FORBES ${ }^{1}$, GRAEME C. HOCKING ${ }^{2}$ and GRAEME A. CHANDLER ${ }^{1}$
}

(Received 1 November 1993; revised 27 July 1994)

\begin{abstract}
Withdrawal flow through a point sink on the bottom of a fluid of finite depth is considered. The fluid is at rest at infinity, and a stagnation point is present at the free surface, directly above the point sink. Numerical solutions are computed by means of the method of fundamental solutions, and it is observed that flows of this type are apparently possible for Froude number less than about 1.5. Relationships to previous work are discussed.
\end{abstract}

\section{Introduction}

In recent years, the problem of determining flow into a point or line sink beneath the free surface of an otherwise stagnant fluid has generated much interest. The problem is of importance in reservoir engineering, for example, since its solution may provide information about optimum withdrawal rates in a variety of circumstances [11]. In the absence of fluid viscosity, it is a simple matter to give a mathematical formulation of this withdrawal problem, and it is at first tempting to suppose that its solution is likewise a straightforward and uncomplicated affair. In some sense, then, withdrawal flow might properly be regarded as a canonical problem in free-surface fluid mechanics, having a simple mathematical statement and often only involving a single physical parameter, but the difficulties introduced by the nonlinearity of the free-surface condition have given some very unexpected outcomes.

Two-dimensional flow into a line source or sink beneath the surface of a fluid of infinite depth was apparently first considered by Peregrine [17]. His work was greatly extended by Tuck and Vanden-Broeck [20], who found an isolated solution with a cusp on the free surface directly above the sink, when the Froude number $F_{2 D}^{\infty}$ (based on the sink submergence depth in a fluid of infinite depth) had the value $F_{2 D}^{\infty}=3.56$.

\footnotetext{
${ }^{1}$ Dept. of Math., The University of Queensland, QLD 4072, Australia.

${ }^{2}$ Dept. of Math., The University of Western Australia, Nedlands, Western Australia 6009, Australia.

(C) Australian Mathematical Society, 1996, Serial-fee code 0334-2700/95
} 
They also reported solutions possessing a free-surface stagnation point above the sink, in the approximate parameter range $F_{2 D}^{\infty}<2$, although no profiles were shown due to the unreliability of the numerical solution in this case. It is natural to suppose that the solutions possessing a stagnation point might correspond to a source beneath the surface, and that the isolated cusped solution at $F_{2 D}^{\infty}=3.56$ could be the result of the presence of a sink, but since the free-surface condition only involves the square of the fluid velocity, there is no distinction in the mathematical formulation between a source or sink, and this appears to be confirmed in the experiments of Imberger [10]. Recently, a third type of solution has been computed by Mekias and Vanden-Broeck [14], and involves a jet-like region near the source, separated by two vortex sheets from the remaining stationary fluid.

Hocking and Forbes [8] have reconsidered the stagnation point solutions of Tuck and Vanden-Broeck [20], and in a careful numerical investigation, they determined that such flows are apparently confined to the interval $0<F_{2 D}^{\infty}<1.42$. No obvious physical mechanism exists to explain the failure of this solution branch at $F_{2 D}^{\infty}=1.42$, although Forbes and Hocking [4] have suggested that the physical solution branch is ultimately limited by the presence of a singularity at this point; this conclusion was reached after a consideration of the effects of surface tension upon the solutions.

Two-dimensional flow into a line sink has also been studied in the case when the water is of finite depth. Numerical solutions were obtained by Hocking [5] for various conditions on the bottom boundary, and were later extended to include the case of infinite Froude number [6]. It is now known that, for finite-depth water, cusped solutions may exist for all Froude numbers $F_{2 D}>1$, when the sink is placed on the fluid bottom (see Hocking [7]); in these flows, the Froude number $F_{2 D}$ is now based on the undisturbed fluid depth, rather than the source immersion depth as above. Flows with a free-surface stagnation point are also possible in finite depth, and appear from the work of Hocking and Forbes [9] to be confined to the approximate interval $0<F_{2 D}<0.3$, with an unexplained failure of the solution branch occurring once again, at about $F_{2 D} \approx 0.3$ when the source is placed on the bottom. Hocking and Forbes [9] were unable to determine whether a steady solution exists in the interval $0.3<F_{2 D}<1$; in particular, a search for free-surface waves in this parameter region was unsuccessful, although waves in this type of flow have been reported by Mekias and Vanden-Broeck [13].

Some work involving three-dimensional fluid flow into a point source or sink has been reported recently. Forbes and Hocking [3] considered fluid of infinite depth, and showed that solutions with a stagnation point on the free surface exist in an interval of Froude numbers $F^{\infty}<6.4$. (This three-dimensional Froude number again uses sink submergence depth as the reference length, since the fluid is of infinite depth.) In this case, the failure of the solution branch at $F^{\infty}=6.4$ has a clear physical explanation, and is associated with a (circular) secondary stagnation line at the free surface, at which 
a type of wave-breaking occurs. This work has since been generalized by Singler and Geer [19] to include a two-layer flow with surface tension, and by Miloh and Tyvand [16], who considered unsteady effects by means of a Taylor-series expansion in time, and reported some agreement with experiment.

The aim of the present paper is to extend the infinite-depth investigations of Forbes and Hocking [3] to the case when the fluid has finite depth. Initially, this seemed as if it should be a very simple task, since it is merely required to add a term to the integral equation used in [3], and the intention was then to consider more elaborate draining flows. However, we have found that the use of an integral-equation formulation, although extremely successful in the infinite-depth case, fails completely when the fluid has finite depth. The reason for this failure is still unclear, but appears to be related to the distribution of the eigenvalues of the nonlinear integral operator in the complex plane, and investigations of this matter are in progress. From the pragmatic perspective of obtaining a reliable numerical solution to this problem, it has therefore proved necessary to develop an alternative solution scheme, and this is described in Section 2. The results are presented in Section 3, and show greater qualitative similarity to the two-dimensional line-sink solutions obtained by Hocking and Forbes [8], than to the three-dimensional results of Forbes and Hocking [3] for a point sink in infinitely deep fluid.

\section{The mathematical formulation}

We consider a stagnant fluid of finite depth $H$ and infinite lateral extent, subject to the downward acceleration $g$ of gravity. A cartesian coordinate system is located in the fluid, so that the $x-y$ plane lies along the horizontal bottom, and the $z$-axis points vertically. The fluid surface therefore occupies the plane $z=H$.

A point sink is now introduced on the bottom of the fluid, at the origin of the coordinate system. It consumes fluid at the volume rate $m$, producing a local flow and disturbing the free surface of the fluid.

Nondimensional variables are defined, using the far-field fluid depth $H$ as the reference length, and the quantity $\sqrt{g H}$ as the unit of speed. Solutions are then dependent only on the single parameter $F=m / \sqrt{g H^{5}}$, which is the (three-dimensional) depth-based Froude number for the flow.

Since the flow geometry is expected to be axisymmetric, we introduce cylindrical polar coordinates $(r, \theta, z)$ by means of the usual relations $x=r \cos \theta$ and $y=r \sin \theta$. In the absence of viscosity, density stratification and initial circulation, the fluid must flow irrotationally, so that the fluid velocity vector $q$ may be expressed as the gradient of a scalar potential function $\Phi(r, z)$. The incompressibility of the fluid is expressed by 
the vanishing of the divergence of the velocity $\mathbf{q}$, which results in Laplace's equation

$$
\nabla^{2} \Phi=\Phi_{r r}+\frac{1}{r} \Phi_{r}+\Phi_{z z}=0
$$

for the velocity potential $\Phi$. The subscripts in (1) denote partial differentiation.

The vanishing of the velocity component normal to the bottom surface $z=0$ gives

$$
\Phi_{z}=0 \quad \text { on } z=0,
$$

except near the point sink at the origin, where instead

$$
\Phi \rightarrow \frac{F}{2 \pi} \frac{1}{\sqrt{r^{2}+z^{2}}} \quad \text { as }(r, z) \rightarrow(0,0) .
$$

At the unknown free surface location $z=\zeta(r)$, there are two constraints to be satisfied. The first of these is a kinematical condition, arising from the fact that no flow occurs normal to the surface, and is expressed in the form

$$
\Phi_{z}=\Phi_{r} \zeta^{\prime}(r) \quad \text { on } z=\zeta(r) .
$$

The second is a dynamical condition stating that the fluid pressure at the free surface must equal the (constant) atmospheric pressure; using Bernoulli's equation in the fluid, this yields the condition

$$
\frac{1}{2}\left(\Phi_{r}^{2}+\Phi_{z}^{2}\right)+z=1 \quad \text { on } z=\zeta(r) .
$$

The difficulty with this problem arises, in part, from the fact that the velocity potential $\Phi(r, z)$ is singular near $(r, z)=(0,0)$ and decays only slowly (in fact $\mathrm{O}(1 / r))$ as $r \rightarrow \infty$. We deal with this by writing

$$
\Phi(r, z)=S(r, z)+\chi(r, z),
$$

where the singular part $S(r, z)$ is the velocity potential due to a sink placed at the origin $(r, z)=(0,0)$, in the presence of parallel plates at $z= \pm 1$. This function also models the behaviour of $\Phi(r, z)$ as $r \rightarrow \infty$. The function $\chi(r, z)$ is now smooth near the origin, and decays faster than $\mathrm{O}(1 / r)$ as $r \rightarrow \infty$, and will therefore be easier to approximate numerically. By the method of images [15], it follows that the singular part $S(r, z)$ may be represented by the series

$$
S(r, z)=\frac{F}{2 \pi}\left[\frac{1}{\sqrt{r^{2}+z^{2}}}+\sum_{\substack{k=-\infty \\(k \neq 0)}}^{\infty} \frac{1}{\sqrt{r^{2}+(z-2 k)^{2}}}-\frac{1}{2|k|}\right] .
$$


This is a combination of the physical sink at the origin $(r, z)=(0,0)$, as required by (3), and an infinite sequence of image sinks at the points $(r, z)=(0, \pm 2 k)$, $k=1,2,3, \ldots$ In fact, we only require the derivatives of $S$ with respect to $r$ and $z$, which are given by

$$
\begin{aligned}
& S_{r}(r, z)=U^{S}(r, z)=-\frac{F}{2 \pi} \sum_{k=-\infty}^{\infty} \frac{r}{\left[r^{2}+(z-2 k)^{2}\right]^{3 / 2}}, \\
& S_{z}(r, z)=W^{S}(r, z)=-\frac{F}{2 \pi} \sum_{k=-\infty}^{\infty} \frac{(z-2 k)}{\left[r^{2}+(z-2 k)^{2}\right]^{3 / 2}} .
\end{aligned}
$$

The series (8) for the singular-part velocities $U^{S}$ and $W^{S}$ are clearly convergent, and the presence of the term $1 / 2|k|$ in (7) guarantees the convergence of this sum also.

The regular part $\chi(r, z)$ of the velocity potential in (6) also satisfies Laplace's equation (1) and the bottom condition (2). The kinematic condition (4) is now written

$$
\left(W^{S}+\chi_{2}\right)=\left(U^{S}+\chi_{r}\right) \zeta^{\prime}(r) \quad \text { on } z=\zeta(r),
$$

and the Bernoulli condition (5) becomes

$$
\frac{1}{2}\left[\left(U^{S}+\chi_{r}\right)^{2}+\left(W^{S}+\chi_{z}\right)^{2}\right]+z=1 \quad \text { on } z=\zeta(r) .
$$

It remains to choose an appropriate representation for the regular-part function $\chi(r, z)$ in (6). One obvious possibility is to make use of an integral equation, as in Forbes and Hocking [3], but we find that the relevant equation is so poorly conditioned that it is of no practical use. This unexpected behaviour is a consequence of the assumption of finite-fluid depth, which clearly has an adverse effect upon the spectrum of the nonlinear integral operator. An alternative to integral-equation techniques is provided by the method of fundamental solutions, in which the function is represented as a distribution of singularities placed outside the region of interest. The technique is described by Bogomolny [1], Mathon and Johnston [12] and Reichel [18] for example, and further references may be found in Chandler and Forbes [2].

In view of the axial symmetry of the problem, we represent the function $\chi(r, z)$ as an integral distribution of ring sinks, of unknown strength $M$, placed at some position $z=\xi$ above the free surface of the fluid. This automatically guarantees the satisfaction of Laplace's equation (1) for the regular-part potential $\chi$, and an image distribution of ring sinks is also placed at $z=-\xi$ in order to satisfy the bottom condition (2) identically. We therefore assume that

$$
\begin{aligned}
\chi(r, z)=\int_{0}^{\infty} M(\rho) \int_{-\pi}^{\pi} & {\left[\frac{1}{\sqrt{r^{2}+\rho^{2}-2 r \rho \cos \beta+(z-\xi)^{2}}}\right.} \\
& \left.+\frac{1}{\sqrt{r^{2}+\rho^{2}-2 r \rho \cos \beta+(z+\xi)^{2}}}\right] d \beta d \rho .
\end{aligned}
$$


In general, the location $\xi$ of the ring sink is allowed to be a smoothly varying function of radius $\rho$, in order to improve the conditioning of the algorithm, and so to enhance its usefulness. For low Froude numbers, it is sufficient to set $\xi=1.1$, but a better distribution is often required as $F$ is increased, and in these cases, a typical choice for the function $\xi$ is $\xi=\zeta+0.05$, in which $\zeta$ is the free-surface elevation.

Once again, it is only the first derivatives of the function $\chi$ that are required, and after some considerable algebra, these may be expressed in the form

$$
\begin{aligned}
& \chi_{r}(r, z)=-\int_{0}^{\infty} M(\rho)\left[\mathbb{K}\left(r, \rho, P^{(-)}, Q\right)+\mathbb{K}\left(r, \rho, P^{(+)}, Q\right)\right] d \rho, \\
& \chi_{z}(r, z)=-\int_{0}^{\infty} M(\rho)\left[(z-\xi) \mathbb{L}\left(P^{(-)}, Q\right)+(z+\xi) \mathbb{L}\left(P^{(+)}, Q\right)\right] d \rho,
\end{aligned}
$$

in which the kernel functions $\mathbb{K}$ and $\mathbb{L}$ involve complete elliptic integrals $K$ and $E$, of the first and second kinds, respectively. They may be derived using the transformations given in the Appendix of Forbes and Hocking [3], and can be written

$$
\begin{aligned}
\mathbb{K}(r, \rho, P, Q) & =\frac{4}{Q \sqrt{P+Q}}\left[\rho K\left(\frac{2 Q}{P+Q}\right)+\frac{r Q-\rho P}{P-Q} E\left(\frac{2 Q}{P+Q}\right)\right], \\
\mathbb{L}(P, Q) & =\frac{4}{(P-Q) \sqrt{P+Q}} E\left(\frac{2 Q}{P+Q}\right),
\end{aligned}
$$

where we have also defined the auxiliary functions

$$
P^{( \pm)}=r^{2}+\rho^{2}+(z \pm \xi)^{2} \quad Q=2 r \rho .
$$

The numerical solution of this highly nonlinear free-surface problem is now reasonably straightforward. Mesh points are chosen at equally spaced values of the radius, $0=r_{1}, r_{2}, \ldots, r_{N}$, and a guess is made for the two functions $\zeta(r)$ and $M(r)$, represented as point values $\zeta_{1}, \zeta_{2}, \ldots, \zeta_{N}$ and $M_{1}, M_{2}, \ldots, M_{N}$ respectively. The free-surface slopes $\zeta_{1}^{\prime}, \zeta_{2}^{\prime}, \ldots, \zeta_{N}^{\prime}$ are obtained with very high accuracy by differentiation of a cubic spline fitted to the estimate for the surface elevation $\zeta(r)$. The singular-part velocities are computed from (8) and the regular-part velocities are obtained from (12) using accurate cubic-spline quadrature. A damped Newton's method is then used to adjust the initial estimate for $\zeta(r)$ and $M(r)$ iteratively, until the kinematic condition (9) and the Bernoulli equation (10) are satisfied at the mesh points.

\section{Discussion of results}

The present study of stagnation-type flow into a point sink on the bottom of a region of fluid of finite depth has much in common with two-dimensional flow into a line sink 
studied by Tuck and Vanden-Broeck [20] and Hocking and Forbes [8]. Specifically, it is found that, for small values of the Froude number $F$, the numerical method has no difficulty finding a highly converged solution to the governing equations. The free surface thus obtained rises to the stagnation height $z=1$ at the point $r=0$, directly above the sink, and there is then a small dip in the surface elevation $\zeta(r)$, at a radius of approximately $r=0.8$. As $r$ increases, the surface rises slowly to the stagnation level $z=1$, consistently with the fact that there can be no fluid velocity at infinity, since the mass flux produced by a point sink is finite.

As the Froude number $F$ is increased, more numerical mesh points are needed at the free surface, to maintain the accuracy of the scheme. We find that, for $F=1.5$, it is required to take at least $N=241$ points, and since the method demands that both the surface elevation $\zeta(r)$ and the sink-strength function $M(r)$ are to be obtained, the Newton algorithm therefore involves 482 variables. A solution at this value of the Froude number is thus obtained only with substantial computational resources.

When we attempted to find a solution at $F=1.51$, using a highly converged solution for $F=1.5$ as an initial guess, the numerical solution algorithm failed. With $N=241$ mesh points, it has been possible to obtain numerical solutions in the interval $1.55<F<1.6$, but for these values, the surface profile contains spurious waves of small wavelength and amplitude. This is precisely the situation described by Tuck and Vanden-Broeck [20] and Hocking and Forbes [8] for two-dimensional flow into a line sink, and in the present paper, we likewise find that the small free-surface waves are an artifice of the numerical method only. More significantly, we find that the determinant of the Newton solution algorithm actually changes sign at about $F=1.5$. On this basis, we are prepared to suggest that the branch of physical solutions only exists in the approximate interval $0<F<1.5$, and that the numerical method then undergoes a bifurcation to a spurious branch of nonphysical solutions for $F>1.5$. This additional branch becomes more difficult to detect as the number $N$ of mesh points is increased.

The numerical evidence therefore suggests that flow involving a free-surface stagnation point with a point sink at the bottom is only possible for $F<1.5$ in fluid of finite depth. The branch of solutions appears to be limited by a singularity at about $F=1.5$, exactly as suggested by Forbes and Hocking [4] for the case of two-dimensional flow into a line sink.

Figure 1 shows two free surface elevations, one of which has been obtained with a moderate Froude number $F=1$ (sketched with a dashed line), and the solution drawn with a solid line corresponds to the maximum Froude number $F=1.5$. Some spurious waves of very small amplitude and wavelength are actually present in the surface profile obtained with $F=1.5$, although they may be too small to be seen on the graph. These solutions are axially symmetric, and this is highlighted by Figure 2 , in which a portion of the full surface profile for the maximum Froude number $F=1.5$ 


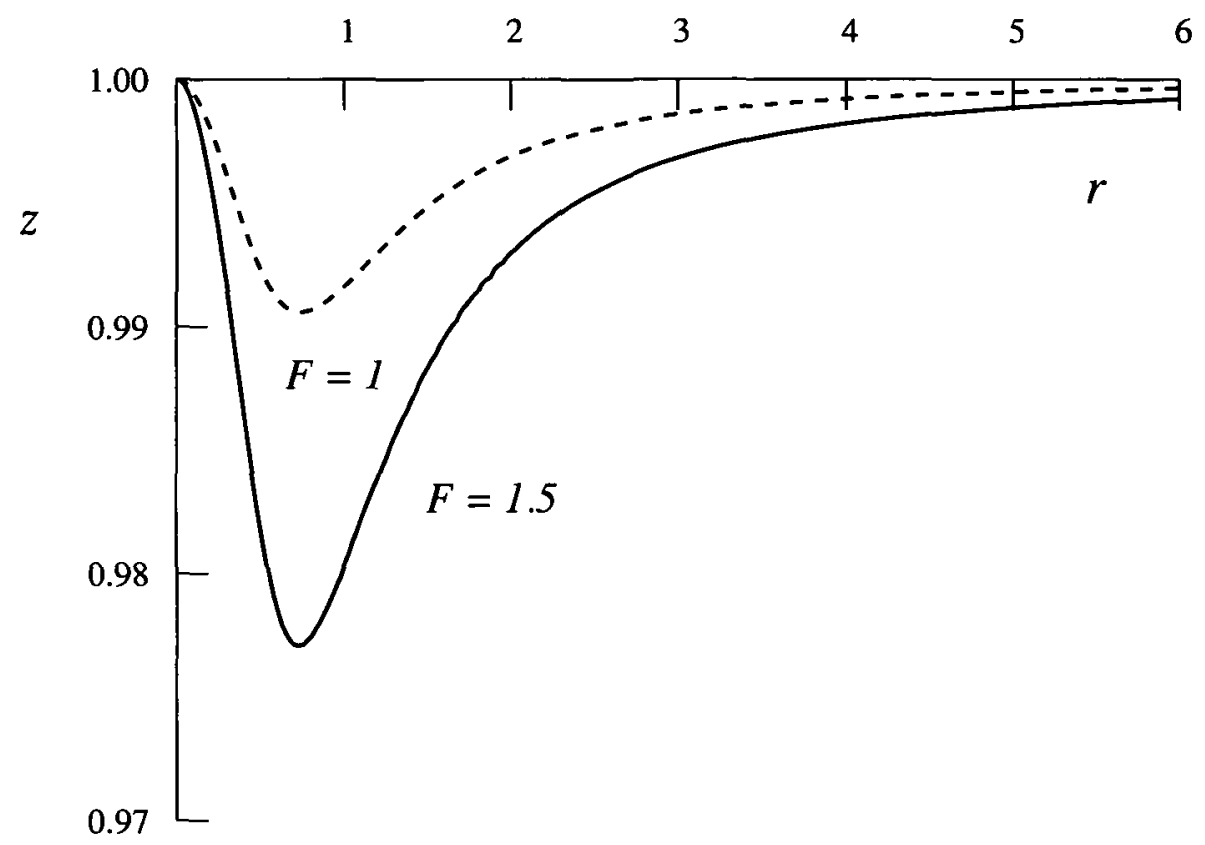

FIGURE 1. Two free-surface profiles, obtained with $F=1$ (dashed line) and $F=1.5$ (solid line).

is shown in perspective. In this diagram, hidden portions of the surface have not been shown, so as to avoid the diagram becoming excessively cluttered.

\section{Conclusions}

Flow caused by the presence of a point sink on the bottom of an otherwise stationary fluid of finite depth has been investigated, using the method of fundamental solutions. It has been found that flow with a free-surface stagnation point can only be determined for Froude numbers in the approximate interval $0<F<1.5$, and the numerical method fails to yield a meaningful solution beyond $F=1.5$.

The height $\zeta_{\min }$ of the lowest free-surface point (at the trough) has been monitored as it varies with Froude number $F$, and the results are shown in Figure 3. No immediate physical explanation is evident for the failure of the numerical solution technique at about $F=1.5$, and we suggest that a mathematical singularity forms near this parameter value, analogously to the two-dimensional work of Forbes and Hocking [4]. 


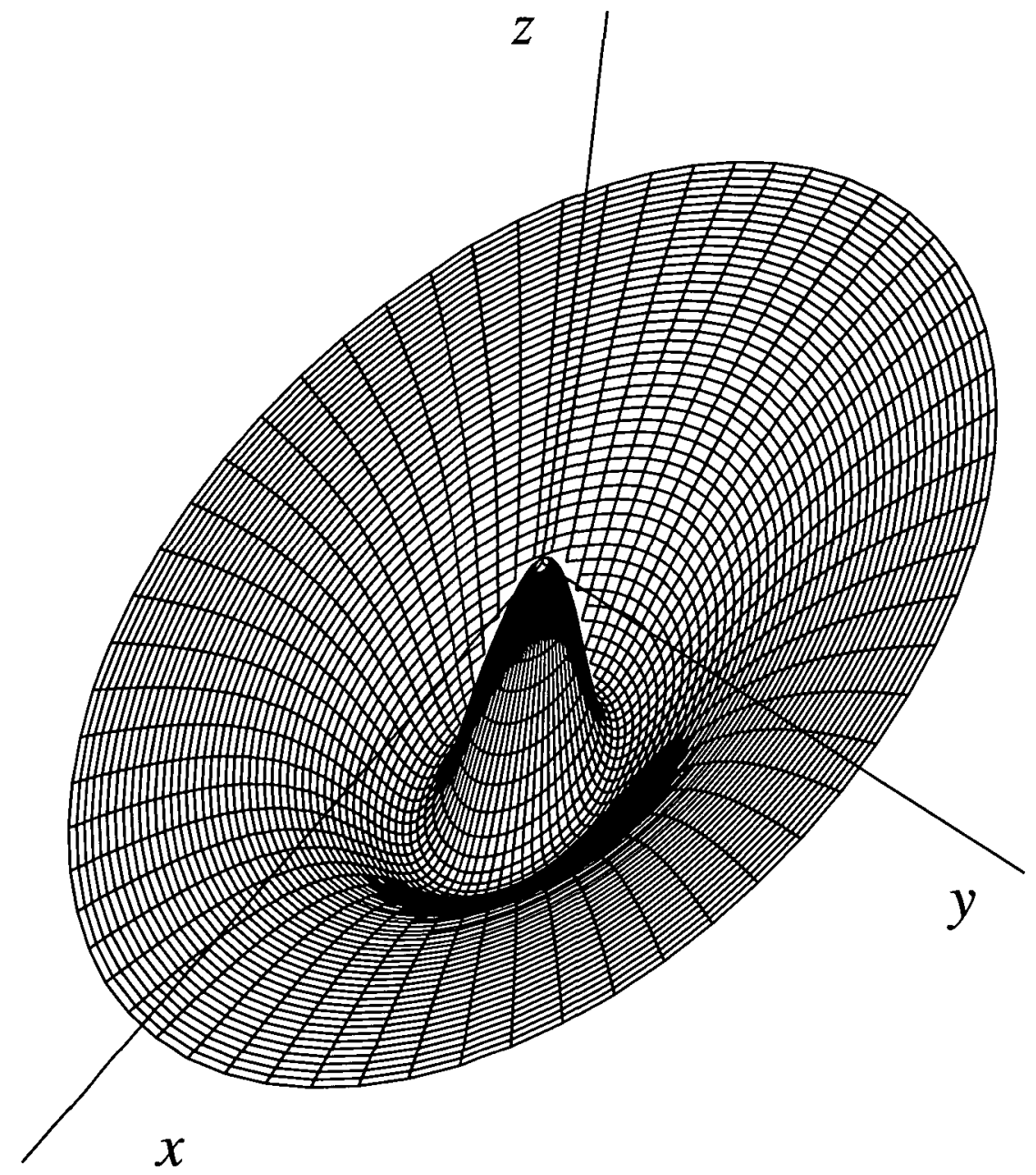

FIGURE 2. Perspective view of the free surface, for the solution with $F=1.5$. The scale on the vertical axis has been magnified by a factor of 100 , and the origin of the coordinate system moved to the stagnation point, for ease of viewing. The surface is shown for $0<r<3$.

A physical explanation for the apparent limiting singularity at $F=1.5$ therefore remains elusive, although it is possible that some unsteady effect may become dominant at this Froude number. In addition, it is not yet known whether other steady solution types are possible for $F>1.5$. We have attempted to find a solution having a cusp at the free surface, following Tuck and Vanden-Broeck [20] and Hocking [5], and have developed a modification to the numerical method presented here, so that vertical sections in the surface profile are able to be treated by means of an arclength 


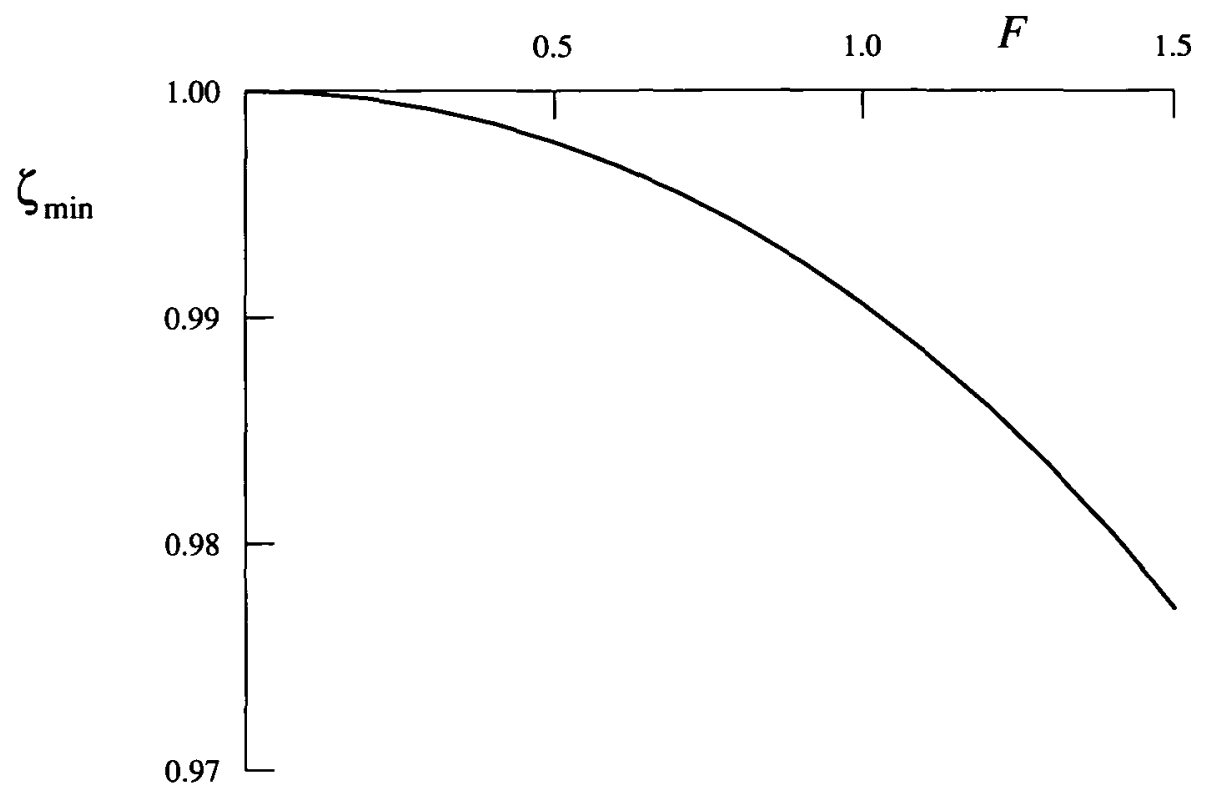

FIGURE 3. The dependence of the minimum surface elevation $\zeta_{\min }$ on the Froude number $F$.

formulation, but no such cusped solutions were found. It is possible that, for large Froude numbers, the surface is drawn down into the sink in some unsteady manner, perhaps involving circulation, and work is currently in progress to compute alternative solutions of this type.

\section{Acknowledgements}

Computing resources for this project were generously provided by the University of Queensland, under enabling grant number R MATHS UEG-7 93. This collaborative work has been made possible by the Ethel Raybould fund at the University of Queensland, and by University of Western Australia Division of Science small grant 133604 , both of which are gratefully acknowledged.

\section{References}

[1] A. Bogomolny, "Fundamental solutions method for elliptic boundary value problems", SIAM J. Numer. Anal. 22 (1985) 644-669. 
[2] G. A. Chandler and L. K. Forbes, "The fundamental solutions method for a free boundary problem", in Proc. 6th CTAC, Canberra, Australia (1994) 122-130.

[3] L. K. Forbes and G. C. Hocking, "Flow caused by a point sink in a fluid having a free surface", $J$. Austral. Math. Soc. Ser. B 32 (1990) 231-249.

[4] L. K. Forbes and G. C. Hocking, "Flow induced by a line sink in a quiescent fluid with surfacetension effects", J. Austral. Math. Soc. Ser. B 34 (1993) 377-391.

[5] G. C. Hocking, "Cusp-like free-surface flows due to a submerged source or sink in the presence of a flat or sloping bottom", J. Austral. Math. Soc. Ser. B 26 (1985) 470-486.

[6] G. C. Hocking, "Infinite Froude number solutions to the problem of a submerged source or sink", J. Austral. Math. Soc. Ser. B 29 (1988) 401-409.

[7] G. C. Hocking, "Critical withdrawal from a two-layer fluid through a line sink", J. Engin. Math. 25 (1991) 1-11.

[8] G. C. Hocking and L. K. Forbes, "A note on the flow induced by a line sink beneath a free surface", J. Austral. Math. Soc. Ser. B 32 (1991) 251-260.

[9] G. C. Hocking and L. K. Forbes, "Subcritical free-surface flow caused by a line source in a fluid of finite depth", J. Engin. Math. 26 (1992) 455-466.

[10] J. Imberger, "Selective withdrawal: a review", in 2nd Intnl Symp. on Stratified Flows, Trondheim, Norway (1980).

[11] G. A. Lawrence and J. Imberger, "Selective withdrawal through a point sink in a continuously stratified fluid with a pycnocline", Univ. of Western Australia, Centre for Water Research, Environmental Dynamics Report ED-79-002 (1979).

[12] R. Mathon and R. L. Johnston, "The approximate solution of elliptic boundary-value problems by fundamental solutions", SIAM J. Numer. Anal. 14 (1977) 638-650.

[13] H. Mekias and J.-M. Vanden-Broeck, "Subcritical flow with a stagnation point due to a source beneath a free surface", Phys. Fluids, Ser. A 3 (1991) 2652-2658.

[14] H. Mekias and J.-M. Vanden-Broeck, "Free-surface flow due to a source submerged in a fluid of infinite depth with two stagnant regions", J. Austral. Math. Soc., Ser. B 34 (1993) 368-376.

[15] L. M. Milne-Thomson, Theoretical hydrodynamics, 5th edition (Macmillan Press Ltd., London, 1979).

[16] T. Miloh and P. A. Tyvand, "Nonlinear transient free-surface flow and dip formation due to a point sink", Phys. Fluids, Ser. A 5 (1993) 1368-1375.

[17] D. H. Peregrine, "A line source beneath a free surface", Univ. Wisconsin Math. Res. Center Tech. Summ. Report 1248 (1972).

[18] L. Reichel, "Solving a model interface problem for the Laplacian operator by boundary collocation and applications", in Proc. of the 3rd European Conf. on Mathematics in Industry (ed. J. Manley et. al), (Kluwer Academic Publishers, Stuttgart, 1990), 119-130.

[19] T. J. Singler and J. F. Geer, "A hybrid perturbation-Galerkin solution to a problem in selective withdrawal", Phys. Fluids, Ser. A 5 (1993) 1156-1166.

[20] E. O. Tuck and J.-M. Vanden-Broeck, "A cusp-like free-surface flow due to a submerged source or sink", J. Austral. Math. Soc. Ser. B 25 (1984) 443-450. 